\title{
Root exudates mediate plant defense against foliar pathogens by recruiting beneficial microbes
}

\author{
Tao Wen ${ }^{1}$, Mengli Zhao ${ }^{1}$, Jun Yuan ${ }^{1,}$, George A. Kowalchuk ${ }^{2}$, Qirong Shen ${ }^{1}$ \\ 1 Jiangsu Provincial Key Lab for Organic Solid Waste Utilization, National Engineering Research Center for Organic-based Fertilizers, Jiangsu \\ Collaborative Innovation Center for Solid Organic Waste Resource Utilization, Nanjing Agricultural University, Nanjing 210095, China \\ 2 Ecology and Biodiversity Group, Department of Biology, Institute of Environmental Biology, Utrecht University, Padualaan 8, 3584 CH \\ Utrecht, The Netherlands
}

\author{
H I G H L I G H T S
}

- Long-chain fatty acids and amino acids application could form foliar disease resistant-soil microbial community.

- Population of Pseudomonas was enriched by long-chain fatty acids and amino acids application.

- The enriched Pseudomonas could help plant resistant foliar pathogens.

\section{ARTICLE INFO}

Article history:

Received February 29, 2020

Revised July 27, 2020

Accepted August 7, 2020

Keywords:

Foliar pathogen resistance

Pseudomonas

Recruitment

Root exudates
GRAPHICAL ABSTRACT

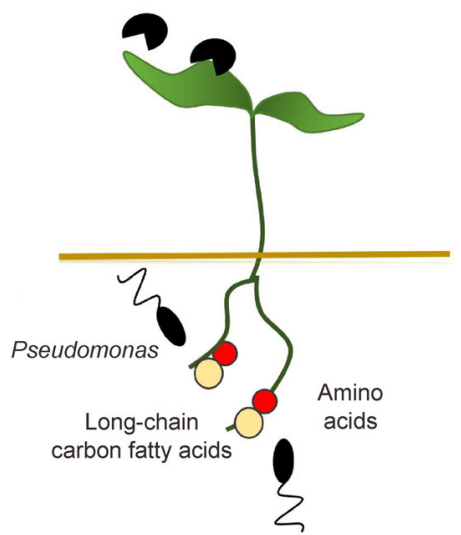

A B S T R A C T

Plants are capable of releasing specific root exudates to recruit beneficial rhizosphere microbes upon foliar pathogen invasion attack, including long-chain fatty acids, amino acids, short-chain organic acids and sugars. Although long-chain fatty acids and amino acids application have been linked to soil legacy effects that improve future plant performance in the presence of the pathogen, the precise mechanisms involved are to a large extent still unknown. Here, we conditioned soils with long-chain fatty acids and amino acids application $(L+A)$ or short-chain organic acids and sugars $(S$ $+S$ ) to examine the direct role of such exudates on soil microbiome structure and function. The $L+A$ treatment recruited higher abundances of Proteobacteria which were further identified as members of the genera Sphingomonas, Pseudomonas, Roseiflexus, and Flavitalea. We then isolated the enriched bacterial strains from these groups, identifying ten Pseudomonas strains that were able to help host plant to resist foliar pathogen infection. Further investigation showed that the $L+A$ treatment resulted in growth promotion of these Pseudomonas strains. Collectively, our data suggest that long-chain fatty acids and amino acids stimulated by foliar pathogen infection can recruit specific Pseudomonas populations that can help protect the host plant or future plant generations.

(c) Higher Education Press 2020

\footnotetext{
* Corresponding author

E-mail address: junyuan@njau.edu.cn (J. Yuan)
}

\section{Introduction}

Plants can impact their associated microbiome as an adaptation strategy when confronted by biotic and abiotic challenges (Toju et al., 2018; Ravanbakhsh et al., 2019). For instance, root exudates can influence the composition and 
function of soil microbial communities (Orwin et al., 2010; Laughlin, 2011; Vries et al., 2012; Grigulis et al., 2013), a phenomenon that is modulated by the quality and/or quantity of available soil nutrients (Bardgett and Wardle 2010). Root exudates can facilitate plant-plant, plant-insect and plantmicrobe interactions (Kessler and Baldwin, 2001; Van Poecke et al., 2001; Kessler and Baldwin, 2002; Rasmann et al., 2005). For example, root exudates and root-derived compounds such as malic acid can recruit specific Bacillus strains to the rhizosphere (Rudrappa et al., 2008); Badri et al. (2009) showed that Arabidopsis ABC transporter mutant (abcg30) which releases more phenolics and there were increased abundance of PGPR or bacteria involved in heavy metal remediation was observed in rhizosphere of Arabidopsis compared to wild type Col-0 plants; recent studies show that $A$. thaliana produces a range of specialized triterpenes that direct the assembly and maintenance of an $A$. thalianaspecific microbiota, enabling it to shape and tailor the microbial community within and around its roots to its own purposes (Huang et al., 2019b).

Interactions between the plant and the rhizosphere soil microbiome in the context of pathogen invasion are generally more specific, with recruitment of specialized microbes potentially antagonistic to a certain pathogen. For example, wheat has been shown to recruit specific Pseudomonas species that produce antimicrobial compounds when confronted with "take-all" disease (Weller et al., 2002). While, beneficial rhizosphere microbes are also able to suppress pathogenesis through the induction of plant induced systemic resistance (ISR), even when the infection did not occur in the rhizosphere. The recruitment of beneficial rhizosphere microorganisms appears to associated with the modification of plant exudation patterns in response to exposure to aboveground pathogens (Yuan et al., 2018). Specifically, long-chain fatty acids and amino acids were identified to play important roles in the recruitment of potentially beneficial microbes (Yuan et al., 2018). However, which groups of the beneficial microbe are and how these compounds recruit them have not yet been elucidated.

In this study, we sought to identify the key microbial taxa enriched in soils amended with rhizosphere cocktails that mimic those produced by plants upon foliar pathogen infection. Furthermore, key bacterial groups were isolated and assayed for their ability to induce foliar pathogen resistance. By combining these approaches, we linked specific exudates classes to the recruitment of bacterial taxa groups that contribute to plant resistance against aboveground pathogen attack.

\section{Materials and methods}

\subsection{Impacts of root exudate classes on the soil microbiome}

To examine the effect of key exudate compounds on the soil microbiome, we selected four categories of different secreted exudates based on our previous results (Yuan et al., 2018).
They are long-chain fatty acids (LCFA) (pentadecanoic acid, hexadecanoic acid, palmitoleic acid, octadecanoic acid, and arachidic acid), amino acids (AA) (isoleucine, leucine, methionine, proline, tryptophan, and ornithine), short-chain organic acids (SCOA) (citric acid, aconitic acid, succinic acid, and malic acid) and sugars (maltose, ribose, glucose, sucrose, fructose, and xylose). In our previous study, LCFA and $A A$ application to soil can increase the plant resistance to foliar pathogen growing in that soil. While this beneficial effect was not seen when applied with SCOA and sugar. The soil conditioning process was as previously described (Yuan et al., 2018). Briefly, for the AA, SCOA, and sugar category, watery solutions were prepared containing each of the selected compounds in equal dosage and to a final total concentration of $10 \mathrm{mM}$. For LCOAs, the total concentration was $10 \mu \mathrm{M}$ due to their lower solubility. The 6-well plates with $15 \mathrm{~g}$ soil in each well were pre-incubated in a growth chamber at $30^{\circ} \mathrm{C}$ for 1 week. Each well then received $1.5 \mathrm{~mL}$ of $L+A$ solution or $S+$ $S$ solution or water twice a week for 8.5 weeks. The $L+A$ solution contains equal volumes of the LCOA and AA solutions, and the $S+S$ solution contains equal volumes of SCOA and sugar solutions. Each treatment consisted of 3 plates with 18 replicates. All plates were randomly placed during the incubation period.

\subsection{DNA extraction PCR amplification and sequencing}

The soil from two wells are randomly pool as one sample, and total soil DNA was extracted from all 27 samples. Extractions were carried out on $500 \mathrm{mg}$ of soil (wet weight) using the Power Soil DNA Isolation kit (Mo Bio Laboratories Inc., Carlsbad, CA, USA), according to the manufacturer's instructions. DNA was extracted from two technical replicates per sample to minimize DNA extraction bias. Samples were stored at $-20^{\circ} \mathrm{C}$, and technical replicates were pooled before performing polymerase chain reaction. DNA quality was assessed according to the $260 / 280 \mathrm{~nm}$ and $260 / 230 \mathrm{~nm}$ absorbance ratios using a NanoDrop ND-2000 spectrophotometer (NanoDrop, ND2000, Thermo Scientific, 111 Wilmington, DE). Bacterial 16S rRNA gene fragments were amplified from the extracted DNA using primers $341 \mathrm{~F}$ (CCTAYGGGRBGCASCAG) and 806R (GGACTACHVGGGTWTCTAAT) following the PCR conditions previously described (Yuan et al., 2018). Amplicons were subjected to 250-bp paired-end sequencing on the Illumina MiSeq sequencing platform at the Genomics Core of Michigan State University.

\subsection{Amplicon sequence processing and analysis}

Amplicon sequences were analyzed using the "DADA2" package in $\mathrm{R}$ environment (version 3.6.1). Initial sequence quality was assessed using the "plotQualityProfile" function. Due to low sequence quality at the 3 '-ends of the reads, joining paired-ends resulted in unreliable reads reducing the number of paired-end reads that passed quality control. We therefore only used the forward reads containing the relatively 
short but highly discriminating V3 region (Callahan et al., 2016). Sequences were truncated at base 120 and trimmed until base 15 . This resulted in relatively short reads ( 120 bp) of high quality from which actual sequence variants (ASVs) were identified.

The DADA2 pipeline produced an ASV count table containing 240000 usable reads and $\sim 2350$ ASVs. Some of them were judged to likely be from chimeric sequences that were not filtered out in the DADA2 pipeline (as a consequence of using only the forward reads) and were thus excluded from further analysis. The resulting final ASV table contained $\sim 200000$ high-quality reads belonging to $\sim 2000$ ASVs. For taxonomy assignment of ASVs, we referenced the protocol of the Earth Microbiome Project (Thompson et al., 2017). Taxonomic assignment of ASVs was accomplished with the assign taxonomy function against the SILVA 16S rRNA database.

\subsection{Selection of bacterial isolates}

The $0.5 \mathrm{~g}$ soil of each treatment was stored in $1 \mathrm{~mL}$ of $5 \mathrm{mM}$ $\mathrm{MgSO}_{4}$ with $25 \%$ glycerol $(\mathrm{v} / \mathrm{v})$ at $-80^{\circ} \mathrm{C}$ at the end of the conditioning experiment for further isolation of beneficial microbes. Before isolation, six replicates of soil samples treated with $L+A$ were thawed at room temperature and suspended by vertexing for $120 \mathrm{~s}$. For the isolation of culturable bacterial species, a serial dilution of each soil sample was plated on $1 / 10$ tryptic soy agar (TSA; Difco) (Rocelle et al., 1995) amended with $200 \mathrm{mg} \mathrm{L}^{-1}$ Delvocid (DSM; active compound: natamycin) to prevent fungal growth and plates were incubated for $3-5$ days at $28^{\circ} \mathrm{C}$. Then, we selected as many as different strains according to morphological characteristics and size of colony, and about $\sim 20$ strains were picked out from each plant. A total of 200 bacterial colonies with unique morphologies were selected and streaked on tryptic soy agar (TSA).

Single colonies from pure cultures were inoculated in $1 / 10$ strength tryptic soy broth (TSB), incubated overnight at $28^{\circ} \mathrm{C}$ at $180 \mathrm{r} \mathrm{min}^{-1}$, and stored at $-80^{\circ} \mathrm{C}$ in $25 \%(\mathrm{v} / \mathrm{v})$ glycerol.

\subsection{Isolate identification}

A loop of bacterial cells was added to $20 \mu \mathrm{L}$ of water, incubated for $15 \mathrm{~min}$ at $95^{\circ} \mathrm{C}$ and immediately cooled on ice. This bacterial lysate was diluted ten times with water and cell debris was removed by centrifugation $(1 \mathrm{~min}, 10000 \times \mathrm{g}$ ). We adopted a one-step barcoded PCR protocol (Goodman et al., 2011), in combination with Illumina sequencing to identify the V5-V7 sequences of bacterial 16S rRNA genes of all isolates. Isolates in 96-well microtiter plates were indexed using the degenerate primers 799F/1193R containing barcodes to amplify the variable regions V5-V7. For PCR amplification, DNA from $1.5 \mu \mathrm{L}$ of lysed cells was amplified using $2 \times$ Premix Taq (Takara Biotechnology, Dalian Co. Ltd., China), $1 \mu \mathrm{L}$ of 0.2 $\mu \mathrm{M}$ forward primer with an 18-bp linker sequence and $1 \mu \mathrm{L}$ of $0.2 \mu \mathrm{M}$ reverse primer (1193R) in a $20 \mu \mathrm{L}$ volume. PCR amplification was performed under the following conditions: $95^{\circ} \mathrm{C}$ for $2 \mathrm{~min}$, followed by 40 cycles of $95^{\circ} \mathrm{C}$ for $30 \mathrm{~s}, 58^{\circ} \mathrm{C}$ for $30 \mathrm{~s}$ and $72^{\circ} \mathrm{C}$ for $30 \mathrm{~s}$, and a final elongation step at $72^{\circ} \mathrm{C}$ for 10 min. PCR products of each 96-well microtiter plate were combined and subsequently purified. Sequences were quality filtered and demultiplexed. OTUs were clustered at $100 \%$ similarity with UNOISE3, which has a built-in error correction to reduce the influence of sequencing errors (Edgar, 2016).

For further identification, the 16S rRNA gene of each isolate was amplified with primers F27/R1492 (Höfle et al., 2005). Two microliters of the colony lysate were added to a total volume of $50 \mu \mathrm{L}$ PCR mixture $(5 \mu \mathrm{L} 10 \times$ Dreamtaq buffer (Thermo Sci-entific), $1 \mu \mathrm{L} 10 \mathrm{mM}$ dNTP's, $2.5 \mu \mathrm{L} 10 \mu \mathrm{M}$ forward primer F27, $2.5 \mu \mathrm{L} 10 \mu \mathrm{M}$ reverse primer $\mathrm{R} 1492,1 \mu \mathrm{L}$ Dreamtaq polymerase (Thermo Scientific), $36 \mu \mathrm{L} \mathrm{H}_{2} \mathrm{O}$. PCR conditions were $5 \mathrm{~min}$ at $94^{\circ} \mathrm{C}$, followed by 30 cycles of $1 \mathrm{~min}$ at $94^{\circ} \mathrm{C}, 1 \mathrm{~min}$ at $55^{\circ} \mathrm{C}$ and $1 \mathrm{~min}$ at $72^{\circ} \mathrm{C}$, and a final extension of $10 \mathrm{~min}$ at $72^{\circ} \mathrm{C}$. PCR products were examined by electrophoresis on $1.5 \%$ agarose gels in $1 \times$ TAE buffer ( 40 mMTris-acetate/1 mM EDTA, pH8) and sequenced by GenScript (Nanjing). Isolate taxonomy was determined through the Sequence Match function of the ribosomal database project (RDP) (Quast et al., 2013). For further exploration of the phylogenetic relationship between enriched ASVs from high throughput sequencing and the isolates, the similarity was showed by alignment the sequence of ASVs to isolates.

\subsection{Microbial induced foliar pathogen resistance assay}

For this assay, 7-day-old Arabidopsis seedlings (Col-0) were prepared and then transplanted into an autoclaved mixture of vermiculite and sand (volume:volume $=1: 1$ ) in 6-well plates, as described previously (Yuan et al., 2018). One week after transplantation, the isolates were pre-cultured in LB broth and applied after re-suspension with autoclaved water at a final concentration of $5 \times 10^{8}$ per well. Autoclaved water was added as the negative control. Then, each tested strain was added to 6 transplanted plants as one replicate and 6 replicates were performed. The test soils were collected from a long-term field experiment that began in January 2014 in the Hengxi town of Nanjing, Jiangsu Province $\left(32^{\circ} 02^{\prime} \mathrm{N}, 118^{\circ} 50^{\prime} \mathrm{E}\right)$. For each pot, $5 \mathrm{~mL}$ of MS medium were added to supply plants with the necessary nutrition during plant growth period. After one week, Pseudomonas syringae pathovar tomato DC3000 (hereafter referred to as Pst) strain was inoculated onto the Arabidopsis leaves as previously described (Yuan et al., 2018). Disease incidence was determined 7 days after infection. Isolates that reduced disease incidence were recognized as beneficial microbes.

2.7 Growth promotion effects of distinct exudate compounds on isolated beneficial strains

Flask culturing was used to test the influence of specific exudate compounds on the growth of the bacterial isolates. Isolates were inoculated in 1/10 strength TSB media, shaken at $170 \mathrm{r} \min ^{-1}$ for $24 \mathrm{~h}$, and cell density adjusted by the 
addition of sterilized water with the final $\mathrm{OD}_{600}$ reached 0.6. Then, $1.5 \mathrm{~mL}$ fresh bacterial suspensions (1\%) were inoculated into $250-\mathrm{mL}$ conical flasks with $150 \mathrm{~mL} 1 / 10$ strength TSB liquid media, and $1.5 \mathrm{~mL}$ of $\mathrm{L}+\mathrm{A}$ or $\mathrm{S}+\mathrm{S}$ solution were added to liquid media with the same concentration as used in soil condition experiment. Controls contained sterile water and same volume of the bacterial suspensions. Cultures were shaken at $170 \mathrm{r} \mathrm{min}^{-1}$ and the absorbance at $\mathrm{OD}_{600}$ was used to determine bacterial growth curves.

2.8 Chemotaxis effects of distinct exudate compounds on isolated beneficial microbes

The modified capillary assay was performed based on the method described by Adler et al. (1973) and our previous publication (Yuan et al., 2015) to quantitatively determine the chemotaxis response of the isolates to the distinct exudate compounds. The strain was grown in LB media until an $\mathrm{OD}_{600}$ of 0.8 was reached. Briefly, the cells were resuspended in the chemotaxis buffer [100 mM potassium phosphate $(\mathrm{pH} 7.0)$ with $20 \mu \mathrm{M}$ EDTA] at concentration of $\mathrm{OD}_{600}=0.8$. A Petri dish $60 \mathrm{~mm}$ in diameter was filled with $10 \mathrm{~mL}$ of the cell suspension prepared above. Standard $1 \mu \mathrm{L}$ capillaries loaded with the exudate cocktails $L+A$ or $S+S$ solution with the same concentration as mentioned above were immersed in the cell suspension in Petri dishes, while the chemotaxis buffer was performed as the negative control. After 30 min incubation in sterile bench, the suspension was then diluted and plated on TSB plates. The CFU were determined by plating on TSB plates and incubating at $37^{\circ} \mathrm{C}$ for $24 \mathrm{~h}$. Each treatment was replicated three times.

\subsection{Statistical methods}

Statistically significant differences $(p<0.05)$ among treatments were evaluated by Student's $t$-test or ANOVA using SPSS. Differences in bacterial community composition across treatments were tested using PERMANOVA (Adonis, transformed data by Bray-Curtis, permutation $=999$ ), implemented in $\mathrm{R}$ version 3.4.3. The DESeq function of the "DESeq2" package (version 1.18.1) (Love et al., 2014) was employed to test for differentially abundant ASVs among conditioned soil samples. Statistical significance was based on $p$-value $<0.05$ (with FDR $<5 \%$ under the Benjamini-Hochberg correction). Statistical analyses of the community of 16S rRNA gene sequencing data were performed using the phyloseq package in the $\mathrm{R}$ environment (version 3.6.1). $\beta$-diversity (PCoA based on Bray-Curtis dissimilarities) was calculated using the "phyloseq" package (version 1.22.3). Statistical significance of the $\beta$-diversity between treatments was calculated through analysis of similarity (adonis), as implemented in the $\mathrm{R}$ environment. The DESeq function of the "DESeq2" package (version 1.18.1) was employed to test for differentially abundant ASVs. Statistical significance was based on $p$ value $<0.05$ (with FDR $<5 \%$ under the Benjamini-Hochberg correction). Graphs of the microbiome data were created using the "ggplot2" package (version 2.2.1).

\section{Results}

3.1 Impact of exudation cocktails on soil bacterial communities

We analyzed the composition of bacterial communities after soil was exposed to two types of exudation cocktails using 16S rRNA gene amplicon sequencing. Average read count per sample was $47705 \pm 8149$. Bacterial communities were highly diverse with the number of ASVs ranging from 480 to 642 per sample in $L+A$ soils (596 \pm 62 ), between 303 and 487 per sample in $S+S$ soils (399 \pm 69 ), with between 313 and 589 per sample in the water/control soils $(461 \pm 76)$. Over all of the groups, the majority of ASVs belonged to the phyla Candidatus_Saccharibacteria $(29.1 \%)$, Proteobacteria (25.6\%), Cyanobacteria (15.7\%), Acidobacteria (6.0\%), Bacteroidetes (5.6\%), and Actinobacteria (7.6\%). Exudate cocktails impacted the composition of the soil bacterial community (Fig. 1A). The $S+S$ treatment significantly increased the relative abundance of Candidatus_Saccharibacteria (from $7.8 \%$ to $62.4 \%$ ), while the $L+A$ addition significantly increased the relative abundance of Proteobacteria (from $20.4 \%$ to $46.1 \%$ ) relative to the control samples. Both treatments decreased the relative abundance of Cyanobacteria from $38.8 \%$ in the control samples to $3.6 \%$ and $4.6 \%$ in the $L+A$ and $S+S$ samples, respectively.

Principal coordinate analysis ( $P C O A)$, based on the detected ASVs, showed a significant difference (PERMANOVA, $p=0.001, R=0.28$ ) in community composition between the two exudates cocktails and the control samples (Fig. 1B). Amino acid and long-chain fatty acid additions increased the alpha-diversity of the soil bacterial community, while the addition of sugars and short chain organic acids decreased the bacterial diversity, as compared with the water control (Fig. 1C).

Compared to the control soils, a total of 22 ASVs were relatively enriched in the $L+A$ samples, yet depleted in $S+S$ samples (Fig. 1D). In order of relative abundance, eight ASVs with relative abundances $>1 \%$ belonged to the genera Sphingomonas, Azohydromonas, Pseudomonas, Roseiflexus, Azoarcus, Flavitalea, and Burkholderia. Previously, it was found that Sphingomonas, Pseudomonas, and Roseiflexus increased in the relative abundance greater in pathogen-conditioned soils (Yuan et al., 2018). In total, $L+A$ exudates influenced general bacterial community composition, while also recruiting a select range of specific bacterial taxa.

3.2 Microbial isolates and their impacts on foliar pathogen resistance

Of the 156 bacterial colonies with unique morphologies selected for further analysis, 23 were putatively identified as belonging to the genera Sphingomonas and Pseudomonas, according to phylogenetic analysis (Fig. 2A). Given the previous link between these general and disease suppression (Yuan et al., 2018), we further examined the 



Fig. 1 (A) Relative abundance (\%) of the major bacterial phyla with significant differences present in the microbial communities of control and exudate-conditioned soil samples. (B) Principal coordinates analysis (PCoA) with Bray-Curtis dissimilarity of the rhizosphere bacterial community in exudates or water conditioned soil. (C) The alpha indices (PD_whole_tree, Chao 1, and Shannon) of rhizosphere bacterial community in each sample. Asterisk indicated the significant difference between two groups. The relative abundance of $22 \mathrm{ASVs}$ that were enriched in the $L+A$ samples, yet relatively depleted in the $S+S$ samples, as compared to the controls. 
ability of these 23 isolates to induce foliar pathogen resistance against foliar infection when applied to plants in sterilized materials. Of these, ten isolates showed an ability to decrease the incidence of disease in our Arabidopsis assay (Fig. 2B). These ten bacteria were all identified as Pseudomonas sp., including Pseudomonas nitroreducens, Pseudomonas plecoglossicida, and Pseudomonas putida with high average nucleotide identities (>99\%) (Fig. 2C).

\subsection{Effects of exudate cocktails on isolate growth}

To further investigate whether the strains with ability to induce foliar pathogen resistance were impacted by the application of specific exudates groups, we examined the ability of exudate application to stimulate growth of the ten strains identified above. The growth of strains $\mathrm{P} 14$ and $\mathrm{P} 90$ was promoted by $\mathrm{L}$ $+A$ yet inhibited by $S+S$ (Fig. 3A). Strains P89 and P137 grew better than the control in response to both $L+A$ and $S+$ $S$ (Fig. $3 A$ ). Strain $P 30$ was only promoted by $L+A$, while strain $P 103$ was inhibited by both $L+A$ and $S+S$ (Fig. 3A). The other four strains (P29, P17, P69, and P34) were not significantly influenced by the exudate's treatments (Fig. 3A).

3.4 Chemotaxis effects of exudate cocktails on the tested isolates

We then tested if the compounds could recruit the isolates. Results indicated that $L+A$ could significantly attract strain P14, P30, P103, and P34, while S + S could only attract P103 and P34 (Fig. 3B). It is also found that the $L+A$ showed higher
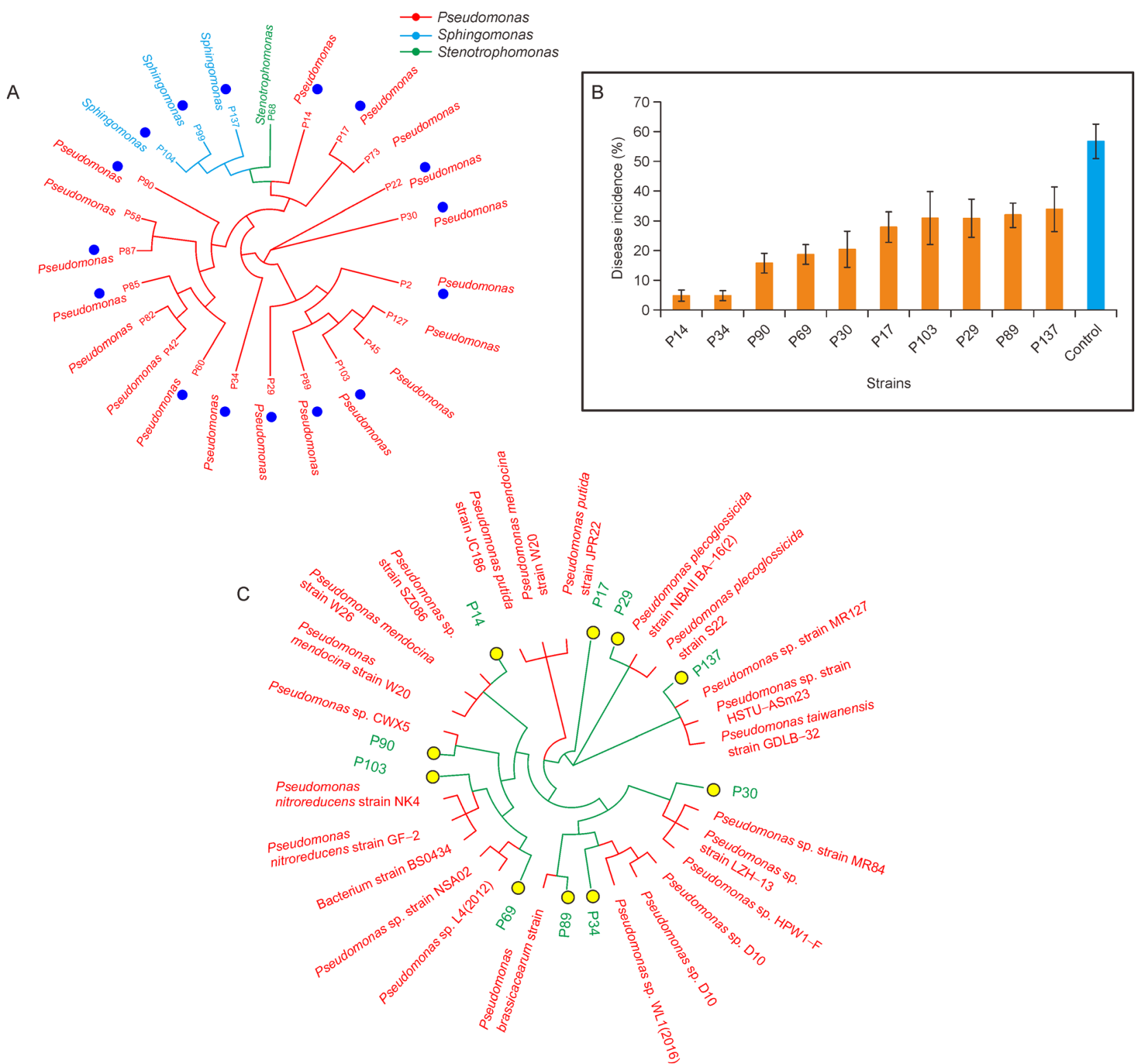

Fig. 2 (A) Maximum-likelihood phylogenetic tree of all 23 representative OTUs clustered with preliminary identification using the highthroughput sequencing method. (B) Foliar pathogen resistance inducement effects of the selected isolates. (C) Phylogenetic tree of the ten beneficial microbe identified as Pseudomonas. 
A

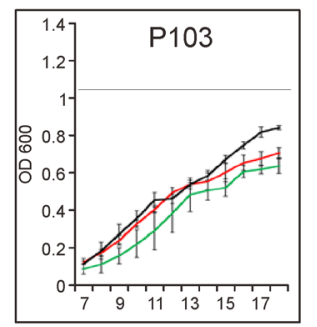

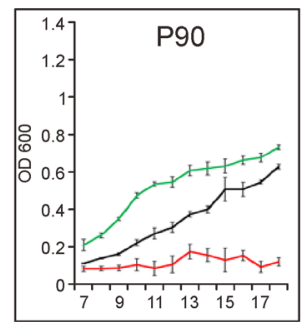

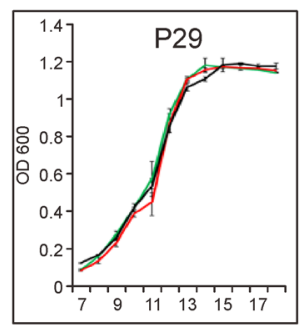

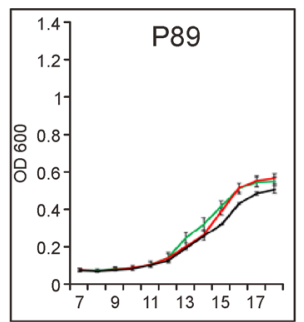

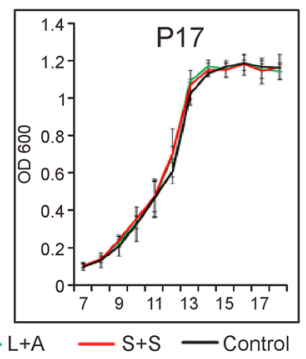

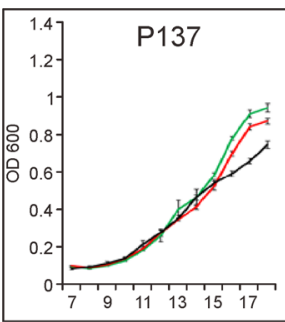

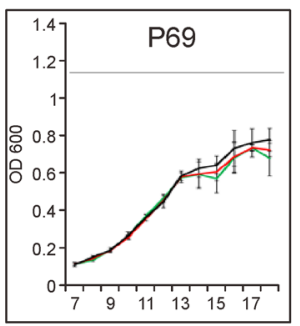

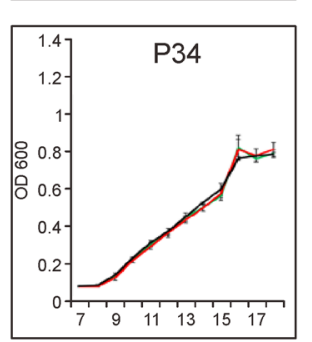

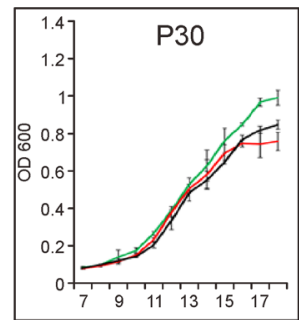

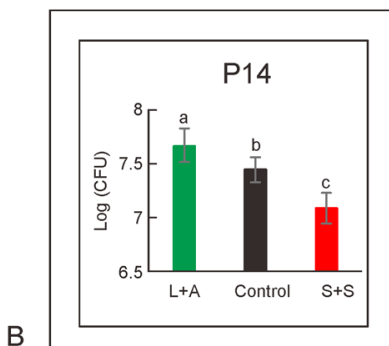


Fig. 3 (A) Effects of two types of exudate cocktails on the growth of 10 tested isolates. (B) Chemotaxis effects of two types of exudate cocktails on the 10 tested isolates Bars indicate the standard errors of the means from three replicates. Columns with different letters are statistically different (ANOVA $p<0.05$ ) among each group.

attraction for strain P90 than S + S (Fig. 3B). Both two types of exudates could not attract strain P89 and P69. Compared with $S+S, L+A$ showed more stronger recruitment effect on the isolates, while $S+S$ showed repellent effect on some isolates such as P14, P90, P30, P29, and P69 (Fig. 3B).

\section{Discussion}

We previously found that foliar pathogen (Pst) infection induced the root secretion of long-chain fatty acids and amino acids, and further found that application of these two groups of compounds could promote host plant systemic resistance, presumably by helping to recruit beneficial microbes (Yuan et al., 2018). Here, we show that addition of long-chain fatty acids and amino acids significantly altered the composition of soil bacterial community by increasing the proportion of Proteobacteria. Conversely, sugars and short- chain organic acids increased the proportion of Candidatus_Saccharibacteria (Fig. 1). We also found that the relative proportion of Sphingomonas, Pseudomonas, Roseiflexus, and Flavitalea increased upon the application of long-chain fatty acids and amino acids, yet decreased in response to application of sugars and short-chain organic acids. These results are in line with our previous data that found higher relative abundances of some of these groups in treatments with foliar pathogen infection (Yuan et al., 2018).

Of foliar pathogen resistance-inducing taxa identified in our study, Pseudomonas has been extensively reported to the ability to induce ISR in a range of plant species (Weller et al., 2007; Pieterse et al., 2014). This genus and Sphingomonas, a bacterial genus closely affiliated with Pseudomonas, were previously reported to be enhanced by the application of longchain fatty acids and amino acids (Kim et al., 2000). After preliminarily identification using high-throughput sequencing 

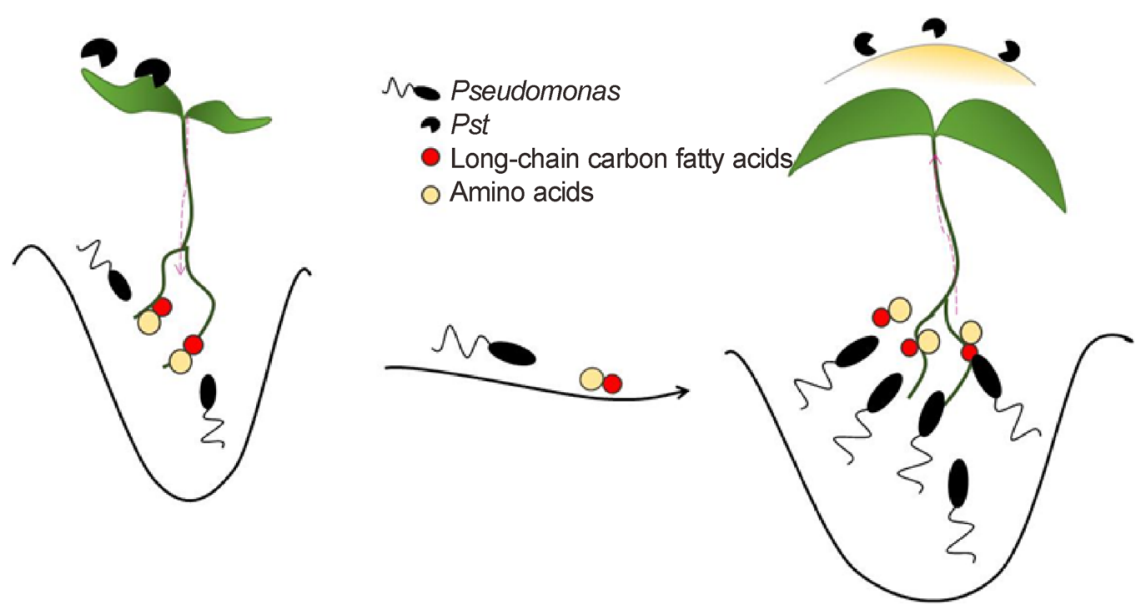

Fig. 4 Mechanistic model showing the plant release of long-chain fatty acids and amino acids to recruit Pseudomonas as a response to foliar pathogens.

with a portion of the 16S rRNA gene, Sphingomonas and Pseudomonas were further studied in the foliar pathogen resistance inducement assay. Ten strains, confirmed to induce foliar pathogen resistance of host plant, were all classified to Pseudomonas (Table 1) and showed $100 \%$ similarity with the ASV8 (Pseudomonas), and only about $80 \%$ similarity with ASV2 (Sphingomonas). This is supported by previous results that host plants can recruit Pseudomonas to their rhizosphere when faced with foliar pathogen attack (Haney et al., 2018). Here, we also make the link between induction of specific classes of exudates to the recruitment of specific taxa capable of inducing foliar pathogen resistance.

It has been found that distinct root exudate compounds influence the cultivation of specific soil microbes. For instance, recent results from Huang et al. (2019a) has reinforced this by demonstrating that terpenes from roots served to cultivate a highly distinct microbial community. Similarly, long-chain fatty acids and amino acids that are over

Table 1 Sequencing alignment of ASV2 and ASV8 to all ten isolates.

\begin{tabular}{lll}
\hline Isolates & Similarity $^{\text {a }}$ (ASV2) & Similarity $^{\mathrm{a}}$ (ASV8) \\
\hline P14 & $100 \%$ & $82 \%$ \\
P34 & $100 \%$ & $82 \%$ \\
P90 & $100 \%$ & $85 \%$ \\
P69 & $100 \%$ & $84 \%$ \\
P30 & $100 \%$ & $82 \%$ \\
P17 & $100 \%$ & $81 \%$ \\
P103 & $100 \%$ & $80 \%$ \\
P29 & $100 \%$ & $80 \%$ \\
P89 & $100 \%$ & $83 \%$ \\
P137 & $100 \%$ & $86 \%$ \\
\hline
\end{tabular}

Note: "a" represented the base matching degree for sequence of ASV2 or ASV8 aligned to isolates. produced under foliar pathogen infection can attract and then promote the growth of some Pseudomonas strains (Fig. 2). Conversely, sugars and short-chain organic acids were shown to exhibit poor beneficial microbe growth promotion and recruitment effects. It should be noted that our study was only able to examine a modest range of strains in this regard, and as such, further research is necessary to determine the full mechanisms underlying the recruitment and growth promotion of this important plant-growth promoting bacterial group.

\section{Conclusion}

In this study, several strains classified as Pseudomonas were identified as beneficial microbe with foliar pathogen resistant inducement and were stimulated by the application of longchain fatty acids and amino acids. These results allow us to complete the whole plant-soil feedback model, which has partly been presented in our previous work (Yuan et al., 2018). Upon foliar pathogen infection, plants release more longchain fatty acids and amino acids to their rhizosphere to recruit and stimulate specific Pseudomonas population. These Pseudomonas populations are then able to induce systemic resistance affording protection from foliar pathogen attack (Fig. 4).

\section{Competing interests}

The authors declare that they have no competing interests.

\section{Acknowledgments}

We thank Ryan Penton (Arizona State University) for the comments on this manuscript. J. Y. was supported by the National Natural Science Foundation of China (31902107), Natural Science Foundation of Jiangsu Province (BK20170724), and National Postdoctoral Program for Innovative Talents (BX201600075). 


\section{Availability of data and materials}

Sequence data are deposited in the NCBI Sequence Read Archive (SRA) database (SRP243322).

\section{Authors' contributions}

JY: performed all experiments; JY and QS designed the study, and wrote the majority of the manuscript; JY, TW and MZ: analyzed the data; GAK: participated in the design of the study, provided comments and edited the manuscript.

\section{References}

Adler, J., 1973. A method for measuring chemotaxis and use of the method to determine optimum conditions for chemotaxis by Escherichia coli. Journal of General Microbiology 74, 77-91.

Bardgett, R.D., Wardle, D.A., (2010). Aboveground-Belowground Linkages: Biotic Interactions, Ecosystem Processes, And Global Change. Oxford University Press.

Badri, D.V. Quintana N., El Kassis, E.G., Kim, H.K., Choi Y.H., Sugiyama, A., Verpoorte, R., Martinoia, E., Manter, D.K., Vivanco, J.M., 2009. An ABC transporter mutation alters root exudation of phytochemicals that provoke an overhaul of natural soil microbiota. Plant Physiol 151, 2006-2017

Callahan, B.J., McMurdie, P.J., Rosen, M.J., Han, A.W., Johnson, A.J. A., Holmes, S.P., 2016. DADA2: high-resolution sample inference from Illumina amplicon data. Nature Methods 13, 581-583.

Edgar, R.C., 2016. UNOISE2: improved error-correction for Illumina $16 S$ and ITS amplicon sequencing. bioRxiv, doi: https://doi.org/ 10.1101/081257.

Goodman, A.L., Kallstrom, G., Faith, J.J., Reyes, A., Moore, A., Dantas, G., Gordon, J.I., 2011. Extensive personal human gut microbiota culture collections characterized and manipulated in gnotobiotic mice. Proceedings of the National Academy of Sciences of the United States of America 108, 6252-6257.

Grigulis, K., Lavorel, S., Krainer, U., Legay, N., Baxendale, C., Dumont, M., Kastl, E., Arnoldi, C., Bardgett, R.D., Poly, F., Pommier, T., Schloter, M., Tappeiner, U., Bahn, M., Clément, J. C., 2013. Relative contributions of plant traits and soil microbial properties to mountain grassland ecosystem services. Journal of Ecology 101, 47-57.

Haney, C.H., Wiesmann, C.L., Shapiro, L.R., Melnyk, R.A., O'Sullivan, L.R., Khorasani, S., Xiao, L., Han, J., Bush, J., Carrillo, J., Pierce, N.E., Ausubel, F.M., 2018. Rhizosphere-associated Pseudomonas induce systemic resistance to herbivores at the cost of susceptibility to bacterial pathogens. Molecular Ecology 27, 1833-1847

Höfle, M.G., Flavier, S., Christen, R., Bötel, J., Labrenz, M., Brettar, I., 2005. Retrieval of nearly complete $16 S$ rRNA gene sequences from environmental DNA following $16 \mathrm{~S}$ rRNA-based community fingerprinting. Environmental Microbiology 7, 670-675.

Huang, A.C., Jiang, T., Liu, Y., Bai, Y.C., Reed, J., Qu, B., Goossens, A., Nützmann, H.W., Bai, Y., Osbourn, A., 2019b. A specialized metabolic network selectively modulates Arabidopsis root microbiota. Science 364, 364 .
Huang, A.C., Jiang, T., Liu, Y.-X., Bai, Y.-C., Reed, J., Qu, B., Goossens, A., Nützmann, H.W., Bai, Y., Osbourn, A., 2019a. A specialized metabolic network selectively modulates Arabidopsis root microbiota. Science, 364, eaau6389.

Kessler, A., Baldwin, I.T., 2001. Defensive function of herbivoreinduced plant volatile emissions in nature. Science 291, 21412144.

Kessler, A., Baldwin, I.T., 2002. Plant responses to insect herbivory: the emerging molecular analysis. Annual Review of Plant Biology 53, 299-328.

Kim, S.J., Chun, J., Bae, K.S., Kim, Y.C., 2000. Polyphasic assignment of an aromatic-degrading Pseudomonas sp., strain DJ77, in the genus Sphingomonas as Sphingomonas chungbukensis sp. nov. International Journal of Systematic and Evolutionary Microbiology 50, 1641-1647.

Laughlin, D.C., 2011. Nitrification is linked to dominant leaf traits rather than functional diversity. Journal of Ecology 99, 1091-1099.

Love, M.I., Huber, W., Anders, S., 2014. Moderated estimation of fold change and dispersion for RNA-seq data with DESeq2. Genome Biology 15, 550.

Orwin, K.H., Buckland, S.M., Johnson, D., Turner, B.L., Smart, S., Oakley, S., Bardgett, R.D., 2010. Linkages of plant traits to soil properties and the functioning of temperate grassland. Journal of Ecology 98, 1074-1083.

Pieterse, C.M., Zamioudis, C., Berendsen, R.L., Weller, D.M., Van Wees, S.C., Bakker, P.A., 2014. Induced systemic resistance by beneficial microbes. Annual Review of Phytopathology 52, 347375.

Quast, C., Pruesse, E., Yilmaz, P., Gerken, J., Schweer, T., Yarza, P., Peplies, J., Glöckner, F.O., 2013. The SILVA ribosomal RNA gene database project: improved data processing and web-based tools. Nucleic Acids Research 41, D590-D596.

Rasmann, S., Köllner, T.G., Degenhardt, J., Hiltpold, I., Toepfer, S., Kuhlmann, U., Gershenzon, J., Turlings, T.C.J., 2005. Recruitment of entomopathogenic nematodes by insect-damaged maize roots. Nature 434, 732-737.

Ravanbakhsh, M., Kowalchuk, G.A., Jousset, A., 2019. Rootassociated microorganisms reprogram plant life history along the growth-stress resistance tradeoff. ISME Journal 13, 30933101.

Rocelle, M., Clavero, S., Beuchat, L.R., 1995. Suitability of selective plating media for recovering heat- or freeze-stressed Escherichia coli O157: H7 from tryptic soy broth and ground beef. Applied and Environmental Microbiology 61, 3268-3273.

Rudrappa, T., Czymmek, K.J., Pare, P.W., Bais, H.P., 2008. Rootsecreted malic acid recruits beneficial soil bacteria. Plant Physiology 148, 1547-1556.

Thompson, L.R., Sanders, J.G., McDonald, D., Amir, A., Ladau, J., Locey, K.J., Prill, R.J., Tripathi, A., Gibbons, S.M., Ackermann, G., Navas-Molina, J.A., Janssen, S., Kopylova, E., Vázquez-Baeza, Y., González, A., Morton, J.T., Mirarab, S., Zech Xu, Z., Jiang, L., Haroon, M.F., Kanbar, J., Zhu, Q., Jin Song, S., Kosciolek, T., Bokulich, N.A., Lefler, J., Brislawn, C.J., Humphrey, G., Owens, S. M., Hampton-Marcell, J., Berg-Lyons, D., McKenzie, V., Fierer, N., Fuhrman, J.A., Clauset, A., Stevens, R.L., Shade, A., Pollard, K.S., Goodwin, K.D., Jansson, J.K., Gilbert, J.A., Knight, R., 2017. A 
communal catalogue reveals Earth's multiscale microbial diversity. Nature 551, 457-463.

Toju, H., Peay, K.G., Yamamichi, M., Narisawa, K., Hiruma, K., Naito, K., Fukuda, S., Ushio, M., Nakaoka, S., Onoda, Y., Yoshida, K., Schlaeppi, K., Bai, Y., Sugiura, R., Ichihashi, Y., Minamisawa, K., Kiers, E.T., 2018. Core microbiomes for sustainable agroecosystems. Nature Plants 4, 247-257.

Van Poecke, R.M., Posthumus, M.A., Dicke, M., 2001. Herbivoreinduced volatile production by Arabidopsis thaliana leads to attraction of the parasitoid Cotesia rubecula: chemical, behavioral, and gene-expression analysis. Journal of Chemical Ecology 27, 1911-1928.

Vries, F.T., Manning, P., Tallowin, J.R., Mortimer, S.R., Pilgrim, E.S., Harrison, K.A., Hobbs, P.J., Quirk, H., Shipley, B., Cornelissen, J. H.C., Kattge, J., Bardgett, R.D., 2012. Abiotic drivers and plant traits explain landscape-scale patterns in soil microbial communities. Ecology Letters 15, 1230-1239.
Weller, D.M., Landa, B., Mavrodi, O., Schroeder, K., De La Fuente, L., Bankhead, S.B., Molar, R.A., Bonsall, R.F., Mavrodi, D.V., Thomashow, L.S., 2007. Role of 2, 4-diacetylphloroglucinolproducing fluorescent Pseudomonas spp. in the defense of plant roots. Plant Biology 9, 4-20.

Weller, D.M., Raaijmakers, J.M., Gardener, B.B., Thomashow, L.S., 2002. Microbial populations responsible for specific soil suppressiveness to plant pathogens. Annual Review of Phytopathology 40, 309-348.

Yuan, J., Zhang, N., Huang, Q., Raza, W., Li, R., Vivanco, J.M., Shen, Q., 2015. Organic acids from root exudates of banana help root colonization of PGPR strain Bacillus amyloliquefaciens NJN-6. Scientific Reports 5, 13438.

Yuan, J., Zhao, J., Wen, T., Zhao, M., Li, R., Goossens, P., Huang, Q., Bai, Y., Vivanco, J.M., Kowalchuk, G.A., Berendsen, R.L., Shen, Q., 2018. Root exudates drive the soil-borne legacy of aboveground pathogen infection. Microbiome 6, 156. 